\title{
LIBRO: EL CURIOSO CASO DE LA ESPECIE SINNOMBRE
}

\section{BOOK: "EL CURIOSO CASO DE LA ESPECIE SIN NOMBRE"}

\author{
Oscar Alberto Flores-Villela ${ }^{1 *}$ \\ ${ }^{1}$ Museo de Zoología, Facultad de Ciencias, , Universidad Nacional Autónoma de México, CDMX, 04510 México. \\ "Correspondence: sapovf@gmail.com
}

Received: 2021-01-13. Accepted: 2021-04-28.

\section{El curioso caso de la especie sin nombre, anécdotas taxonómicas de muy diversos géneros}

Luis Javier Plata Rosas. 2019. Ilustrado por Sebastián Flores. Editorial Universitaria de Guadalajara. 285 pp. Pasta dura.

El autor de este libro, es un oceanógrafo, actualmente miembro del personal académico de la Universidad de Guadalajara que tiene una larga trayectoria en divulgación de la ciencia, teniendo un papel muy destacado en esta área. Este es un libro extraño. No es un libro de taxonomía, aunque tiene información sobre el origen de la clasificación binomial, una muy resumida guía para nombrar especies y algunas reglas muy generales sobre el latín y griego que se usan para nombrar a las especies. También nos habla de la biodiversidad en tres capsulas a lo largo del libro. Nos trata de convencer del valor de la taxonomía, en tres capsulas a lo largo del texto (que no están en el índice), intituladas "La taxonomía según los taxónomos...” Concluyendo con un epitafio a Don Miguel Álvarez del Toro, zoólogo autodidacta, mexicano muy destacado.

El libro presenta una gran variedad de nombres científicos de muchos tipos de especies y acomodados en diferentes categorías, por temas, que reúnen nombres científicos en grupos que organizó, el autor, con mucho ingenio y, seguramente, con muchas horas de investigación. Temas como gobernantes o personajes de la política, a los cuales se les ha dedicado una especie. Como el emperador Hiroito de Japón (Rotaovula hiroitoi, un caracol) o el actual presidente de México, al que se le dedicó un árbol de Los Tuxtlas, Veracruz (Magnolia lopezobradorii). Un género de escarabajo dedicado a Salvador Allende (Allendia).

Nombres dedicados a personajes de películas Yoda purpura (un hemicordado de las profundidaes); al creador de los Simpsom un cangrejo (Albunea groeningi); al creador de Charlie Brown por el personaje de Luci, una especie de cangrejo de arena (Lepidopa luciae). El autor registra nombres dedicados al famoso caricaturista de "The Far Side" Gary Larson (Strigiphilus garylarsoni [ectoparásito de aves] y Serratoterga larsoni [una mariposa]), quien dicho sea de paso, fue alumno de un herpetólogo (H. M. Smith

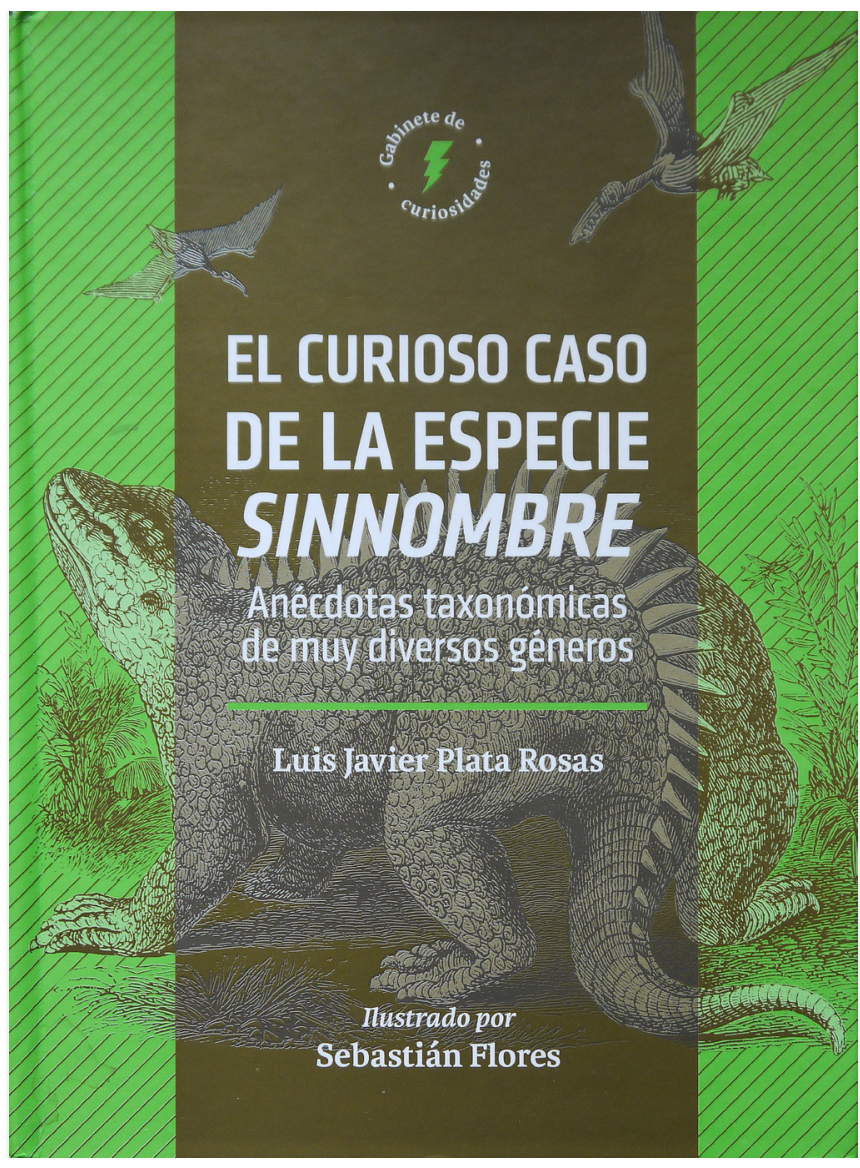

Figure 1. Book cover El curioso caso de la especie sin nombre, anécdotas taxonómicas de muy diversos géneros.

Figura 1. Portada del libro El curioso caso de la especie sin nombre, anécdotas taxonómicas de muy diversos géneros.

com. pers.), y pueden apreciarse sus antecedentes biológicos en muchas de sus caricaturas. En particular yo uso varios de sus chistes para las presentaciones de mis clases en la Facultad de Ciencias de la UNAM.

El autor nos ofrece una reseña de nombres científicos, casi impronunciables por lo largo (52 letras) Gammaracanthuskytodermogammarus loticatobaicalensis (un 
crustáceo, cuyo nombre fue suprimido por la Comisión Internacional de Nomenclatura Zoológica), y otros más que no voy a transcribir. En el desfile de personajes famosos a los cuales se les ha dedicado algún nombre científico está el roquero, muy controversial, Jim Morrison, el Rey Lagarto, así que la lagartija fósil Barbaturex morrisoni es un nombre muy adecuado. Morrison no es el único roquero con un nombre científico en su honor, hay dos ranas del género Dendrosophus, D. ozzy, dedicada a Ozzy Osbourne y D. stingi, dedicada a Sting. No podían faltar nombres dedicados a los Beatles, por ejemplo: Bushelia beatlesi, un poliqueto marino. Autores clásicos tienen sus nombres también, por ejemplo el himenóptero Mozartella beethoveni, pone juntos en el mismo nombre a Mozart y Beethoven. Uno de mis escritores favoritos H. P. Lovecraft, también tiene nombres científicos dedicados a él (Cthulhu) género de protista flagelado. El autor de Parque Jurásico Michael Crichton, tiene un género de dinosaurio dedicado a él, Chrichtonsaurus.

En este libro se cuentan historias de varios nombres de reptiles y anfibios, podríamos empezar con los que tienen nombres repetidos en el género y la especie. La rana Bombina bombina, el sapo Bufo bufo y la salamandra común Salamandra salamandra. Las lagartijas Agama agama, Basiliscus basiliscus; la tortuga marina Caretta caretta y la cobra común Naja naja. Reptiles dedicados a personajes históricos, como Franklin Delano Roosevelt, las lagartijas Anolis roosevelti y Spaherodactylus roosevelti, hay una ranita en Colombia dedicada al príncipe Charles Hyloscirtus princecharlesi. Quizá uno de los naturalistas más homenajeado con nombres es Charles Darwin, existen varios anfibios y reptiles dedicados a este célebre personaje. Entre los anfibios encontramos a Rhinoderma darwinii, que está en peligro de extinción y tiene un modo de reproducción muy interesante, pues los machos después de haber fertilizado los huevos, esperan a que se desarrollen hasta cerca de que los renacuajos estén a punto de eclosionar y los introducen en su boca en donde completan su desarrollo en el saco bucal. Entre los reptiles existen especies dedicadas a Darwin, el anfísbénido Amphisbaena darwini; el gecko Gymnodactylus darwinii, una tortuga Geochelone darwini (ahora Chelonoidis darwini). Pero los dos personajes postulantes de la selección natural están juntos en un nombre de una mosca Wallacea darwini.
También el autor, nos da una orientación de cómo podemos lograr que nos dediquen una especie, siemprey cuando paguemos por ello. Esto puede representar una avenida muy controversial del trabajo taxonómico, pero también tiene alternativas que pueden ayudar a financiar este trabajo de ciencia básica, al cual no se le dedican muchos recursos, por lo menos en los países en vías de desarrollo. Muchos de estos países, también tienen un gran desconocimiento en cuanto a las especies que los habitan, una paradoja.

El libro tiene un índice al principio con los temas por los que agrupa a las especies y un índice de nombres científicos al final, aunque no es alfabético, sino progresivo, como se van citando los nombres en el texto. Me hubiera gustado ver un glosario. Esto haría que público menos especializado que lea este libro entienda varios de los terminajos (lenguaje especializado) de los taxónomos. No faltan algunos pequeños errores editoriales, solo señalo algunos de los que encontré. En la literatura citada hay algunas referencias que no están en orden alfabético en la letra "V". Encontré un error en que el primer nombre del autor de un trabajo está en índice de citas y no está ordenado por su apellido. Cómo escribe el autor "Errare nomenclatorum est" el organismo encargado de aprobar y dar estabilidad a la nomenclatura es la "Comisión Internacional de Nomenclatura Zoológica", no la "Linnean Society".

En resumen, no podría dar ejemplos de todos los nombres que la gran imaginación de los taxónomos han creado, y tampoco podría mencionar a los personajes, hechos y situaciones en las que se diseñaron estos nombres. Independientemente de cual sea la clasificación de los seres vivos (linnenana o el filocode), si los taxónomos se ponen o no de acuerdo, la nomenclatura de las especies ha sido y es un ejercicio de divertimento para los taxónomos. La imaginación de los taxónomos no tiene límites. Luis Javier Plata Rosas, nos ha ahorrado el trabajo de buscarlos y si nos queremos divertir con un poco de ciencia, hay que leer este libro. Lo recomiendo ampliamente. 